\title{
CONFORT PSICOLÓGICO Y EXPERIENCIA TURÍSTICA. CASOS DE ESTUDIO DE ESPACIOS NATURALES PROTEGIDOS DE LA COMUNIDAD VALENCIANA (ESPAÑA)
}

\author{
María José Viñals, Maryland Morant y Lola Teruel \\ Universitat Politècnica de València \\ mvinals@cgf.upv.es, marmogon@cgf.upv.es, dteruel@upvnet.upv.es
}

\section{RESUMEN}

Este trabajo identifica y analiza los factores que configuran el confort psicológico de los visitantes en espacios naturales protegidos y su relación con una experiencia turística satisfactoria. Estos factores han sido analizados en cuatro espacios protegidos de la Comunidad Valenciana (Font Roja, Albufera, Turia e Islas Columbretes) a partir de estudios de observación directa y participante, entrevistas a testigos privilegiados y un total de 2.749 encuestas a visitantes.

Palabras clave: Experiencia turística, Confort Psicológico, Espacios Naturales Protegidos, Comunidad Valenciana.

\section{ABSTRACT}

This paper identifies and analyses the factors setting up the visitors' psychological comfort in natural protected areas and the satisfaction about the tourist experience. These factors have been analyzed in four Valencian Natural Protected Areas (Font Roja, Albufera, Turia and Islas Columbretes) from direct and participant observation, in-depth interviews to key stakeholders, as well as an amount of 2,749 visitors' surveys.

Keywords: Tourist Experience, Psychological Comfort, Natural Protected Areas, Valencian Region.

Fecha de recepción: septiembre 2012.

Fecha de aceptación: septiembre 2013. 


\section{INTRODUCCIÓN}

El confort es un componente determinante de la satisfacción de la visita a un espacio natural protegido ya que influye en cómo el visitante percibe el lugar y la calidad de la experiencia.

El concepto de confort es amplio y se asimila básicamente al de bienestar del individuo en relación al entorno donde se encuentra; es decir, es una expresión del equilibrio entre el hombre y el medio. Según Slater (1985), el confort incluye componentes físicas, fisiológicas y psicológicas. De todas ellas, las físicas y fisiológicas están relacionadas con el medio, por lo que pueden ser más objetivas de valorar y afectan de una manera similar a todos los individuos; pero el confort psicológico presenta una mayor subjetividad ya que cada persona percibe la realidad de forma diferente dependiendo de su perfil socio-demográfico y rasgos de personalidad.

En un espacio natural protegido, el confort psicológico de un visitante se puede asimilar al estado de relajación mental y bienestar que le permite realizar la actividad recreativa prevista en óptimas condiciones sin que nada ni nadie desvíe su atención ni perturbe el normal desarrollo de la misma.

El objetivo principal de este trabajo es identificar y analizar los factores que influyen en el confort psicológico de los visitantes en espacios naturales protegidos con la intención de generar un conocimiento útil para planificadores y gestores que ayude a mejorar la experiencia recreativa de los usuarios. Por tratarse de espacios naturales protegidos, se debe considerar que la experiencia que se brinda al público debe ser auténtica y por tanto será necesario también proporcionar un confort en condiciones que no se desvirtúen los valores de los recursos por los que el sitio fue protegido.

Cada vez son más los trabajos metodológicos llevados a cabo por organismos internacionales en los que se incorpora el grado de satisfacción del turista y donde se observa que ésta tiene una estrecha vinculación con el confort psicológico (World Tourism Organization, 2005). El mundo académico ha analizado también este tema y se puede decir que en estos momentos hay un gran interés entre los investigadores debido a la importancia que se concede a la prestación de una atención de calidad al cliente como garantía de satisfacción de la experiencia recreativa y, en definitiva, de la imagen del destino turístico.

Este trabajo de investigación presenta como innovación una estructura de análisis y valoración del confort psicológico de los visitantes articulado en torno al estudio de una serie de factores. Así, tenemos: factores físico-fisiológicos (especialmente los bioclimáticos y la seguridad); los factores relacionados con el entorno (percepción ambiental), como son el tipo de espacio geográfico o escenario de la actividad y sus atractivos, la actividad recreativa desarrollada y las condiciones en que se organiza (patrón de la visita, grupos, frecuentación, etc.); factores relacionados con los equipamientos y servicios existentes, especialmente los de información e interpretación; y factores que tienen que ver con los individuos como son el perfil sociodemográfico, los rasgos de personalidad, las motivaciones y expectativas y el análisis de su comportamiento ya que éste es el reflejo de las sensaciones y emociones que experimentan los visitantes en el lugar.

Para verificar las hipótesis de trabajo, un total de 2.749 encuestas personales fueron realizadas en temporada alta, entre los años 2004 y 2009, en espacios protegidos de la Comunidad Valenciana (España): Parque Natural del Carrascal de la Font Roja (Ibi y Alcoi, Alicante), 
Reserva Natural de las Islas Columbretes (Castellón), Parque Natural de 1'Albufera de Valencia y Parque Natural del Turia, estos dos últimos en la provincia de Valencia. El cuestionario estaba dividido en: una sección que recogía información personal (género, edad, lugar de residencia, nivel de estudios y tipo de trabajo) para poder definir el perfil socio-demográfico del visitante. La segunda parte planteaba cuestiones sobre las actividades desarrolladas en el parque; y la tercera recababa información sobre las motivaciones, opiniones y grado de satisfacción que los visitantes tenían del espacio protegido. Mientras que las dos primeras partes eran de respuesta cerrada, en la última parte se le permitía al entrevistado expresar libremente sus opiniones. De esta manera se pudo componer una imagen de la percepción, expectativas y experiencia vivida de los visitantes en los espacios naturales visitados.

Los directores de los parques, técnicos, guardas y guías-intérpretes fueron entrevistados, así como miembros de organizaciones no gubernamentales, vecinos, personas con intereses en los parques, prestatarios de servicios, académicos y expertos conocedores de las áreas de estudio. Además, entre 2010 y 2012, se llevaron a cabo diversas sesiones de campo (hasta un total de 98), para la observación del comportamiento de los usuarios (en diversos rangos horarios y días diferentes de la semana). También se realizaron bajo la fórmula de la observación participante, visitas organizadas con grupos escolares y universitarios y también con grupos especializados (ornitólogos, ciclistas, jinetes a caballo, etc.) con el fin de completar y contrastar la información durante el desarrollo del propio acto de la visita.

Respecto a antecedentes de estudios similares, hay que mencionar que el análisis de la experiencia turística en áreas protegidas constituye en la actualidad un tema de gran interés al que cada vez se dedican más trabajos, pero no es muy frecuente encontrar trabajos que aborden de forma integral todos los aspectos que aquí se han analizado; si bien hay que mencionar que hay estudios que analizan elementos individuales de los tratados en este estudio. De esta forma, la innovación que presenta este estudio radica en la visión integrada de análisis de todos estos elementos como componentes básicos del confort y, por ende, de la experiencia turística. Por esta razón, las referencias bibliográficas sobre estudios previos aparecen a lo largo del trabajo y están relacionadas con el elemento concreto abordado en cada apartado.

\section{CONFORT PSICOLÓGICO: FACTORES CLAVE DEL ANÁLISIS}

\subsection{Requerimientos físico-fisiológicos}

El confort psicológico de un visitante en un espacio natural empieza con el cumplimiento de unos requerimientos básicos de tipo físico-fisiológicos como son los bioclimáticos y los de seguridad. A ellos se pueden sumar elementos relacionados con el confort acústico, especialmente relevantes si son espacios naturales donde se espera que no haya ruidos que perturben el paisaje sonoro.

\subsubsection{Factores bioclimáticos}

El clima es especialmente relevante si las actividades se van a realizar al aire libre como es el caso de los espacios naturales. Se trata de uno de los recursos de mayor peso en la valoración turística de un lugar como se desprende del trabajo de Gómez Martín (2005). 
En términos fisiológicos sencillos, la sensación de confort bioclimático puede definirse como la situación en la que nuestro cuerpo hace el menor esfuerzo para regular su temperatura interna. Por ello, se ha recurrido para este análisis a la metodología de Olgyay (1998) que considera tres aspectos básicos en relación al desarrollo de actividades recreativas al aire libre: exigencia de seguridad, de confort climático y salud, y de disfrute. La primera hace referencia a la incompatibilidad del desarrollo de actividades recreativas con una alta frecuencia de riesgos climáticos. La exigencia de un clima saludable tiene que ver con la existencia de condiciones de bienestar en relación a la temperatura (confort térmico) y la humedad relativa (confort higrométrico). La exigencia de disfrute está relacionada con la frecuencia de días soleados y sin lluvia ya que permiten la práctica de actividades al aire libre sin problemas.

\subsubsection{Seguridad}

Los usuarios deben visitar un lugar y desarrollar sus actividades en condiciones de seguridad y, además, deben percibirlo así para su tranquilidad. La necesidad de seguridad se formula entre las más básicas para el ser humano tal y como señaló en su día Maslow (1954) que la situaba en segundo lugar de la jerarquía tras las necesidades fisiológicas. Sönmez (1998) apunta que paz, calma y seguridad son prerrequisitos para atraer turistas a un destino.

Las causas que provocan inseguridad en los visitantes en un espacio natural pueden ser muy diferentes. En primer lugar, hay que mencionar la existencia de riesgos naturales que, por supuesto, no son exclusivos de los espacios naturales protegidos sino que son comunes a cualquier área. Otras causas de inseguridad se relacionan con la carencia o mal estado de ciertos equipamientos e instalaciones. Así, caminos y vallas protectoras en malas condiciones pueden producir sensación de peligro o ser causa real de accidentes. La falta de señalización puede hacer que los visitantes se pierdan y, por ejemplo, la falta de buenas comunicaciones y planes de emergencia pueden dificultar los rescates en casos de accidente. Xuequing Qi et al., (2009) comentan que la seguridad es un importante factor que contribuye a la configuración de la imagen de un destino y afecta la elección del turista respecto a visitar determinados lugares.

Hay que mencionar además que la sensación de seguridad está vinculada a la percepción del riesgo y que ésta varía según los rasgos de personalidad de cada individuo y también con el género, experiencias anteriores, y país de origen, entre otros factores, tal como sugieren Sönmez y Graefe (1998). Lepp y Gibson (2003) apuntan que hay turistas que buscan novedades y retos, los conocidos como «viajeros alocéntricos» según la clasificación de Plog (1974), y también los que buscan sensaciones según Zuckerman (2007) que tienen una percepción del riesgo baja y necesitan estímulos fuertes para alcanzar una experiencia recreativa satisfactoria, por lo que asumen comportamientos arriesgados. De otro lado, están los «turistas psicocéntricos» que buscan viajes donde todo sea previsible y organizado sin dejar nada a la improvisación ya que tienen una percepción del riesgo muy alta y por ello quieren la seguridad que ofrece un viaje bajo control.

Para mitigar los riesgos, es imprescindible que el área protegida esté dotada de un programa de seguridad y emergencias y que los usuarios estén informados de los posibles riesgos y/o limitaciones existentes para la práctica de las actividades recreativas. Por ello, todos estos 
términos deben estar claramente expuestos en forma de recomendaciones en la señalización, en los mapas, folletos y comunicarse a través de los informadores y guías-intérpretes. Un visitante bien informado acerca de las vicisitudes del lugar, lo hace de forma más tranquila, confortable y relajada porque sabe con lo que se va a encontrar y cómo comportarse ante cualquier eventualidad. En España, todas estas recomendaciones se encuentran recogidas en la norma UNE 187002:2008 desarrollada por la Secretaría de Estado de Turismo en la que se establecen los requisitos de nivel de servicio al visitante que debe cumplir un espacio natural protegido, tanto en lo relativo a las instalaciones y equipamientos de uso público, como a todos los procesos necesarios para la prestación de un servicio de calidad (AENOR, 2008).

\subsection{Factores relacionados con el entorno}

\subsubsection{El espacio o escenario donde tienen lugar las actividades recreativas}

Las primeras cuestiones que se plantean en el estudio de este factor consisten en saber qué características tiene ese espacio, de cuánta superficie vamos a disponer para el uso público, y cuánto espacio personal precisará cada visitante para desarrollar la actividad en condiciones de confort.

En cuanto al tipo de espacios, es importante determinar si se trata de espacios abiertos (playas, bosques, praderas, lagos, humedales, etc.), abiertos con barreras físicas (senderos, áreas de pic-nic, etc.) o espacios cerrados (cuevas, ecomuseos, centros de visitantes, etc.), ya que su tipología va a influir decisivamente en las condiciones de confort. En el caso de los espacios abiertos con barreras físicas y en los espacios cerrados, el confort psicológico está muy influido por los fenómenos de saturación y congestión; además, presentan unas limitaciones de aforo en relación al diseño constructivo. El análisis de los espacios abiertos naturales es más complicado, ya que el escenario suele ser mucho mayor y las tipologías de los mismos son muy diversas como hemos visto, por lo que las variables a tratar cambiarán mucho de un ecosistema a otro. De otro lado, es necesario conocer la zonificación del área protegida, pues es evidente que muchos espacios de alto valor ecológico se preservarán del uso público y solamente una parte del territorio quedará disponible para llevar a cabo actividades recreativas.

El nivel de frecuentación del espacio protegido debe ser el adecuado para garantizar la conservación y protección de los recursos y aquel con el que los usuarios se sientan cómodos. Un nivel de frecuentación elevado es siempre un problema y provoca saturación generalizada y/o congestión puntual en determinados lugares o equipamientos. La literatura científica turística ha dedicado muchos trabajos a este tema (Manning, 1999; Morgan y Lok, 2000; Vaske y Donnelly, 2002; World Tourism Organization, 2004) haciendo alusión a la insatisfacción de la experiencia recreativa que estos fenómenos llevan aparejada a causa del disconfort psicológico que producen en aquellos visitantes que buscan especialmente ambientes donde disfrutar la naturaleza en soledad. No obstante, otros visitantes, con rasgos de personalidad diferentes, pueden percibir negativamente espacios poco frecuentados, como es el caso de personas con un marcado carácter agorafóbico, los cuales pueden encontrar en los espacios naturales lugares muy propicios para desencadenar sensaciones de inseguridad y peligro (Davidson, 2003; Bernarda y Lewis, 2003; Van Tilburg y Vingerhoets, 2005; Vivas, 2009). Marion y Farrell (1998) 
se han preocupado en identificar toda una serie de estrategias para mitigar la percepción de saturación y congestión como son: modificar las áreas de uso intensivo, cambiar el patrón de la visita, etc. Hay que decir que estas medidas de carácter corrector tienen un efecto limitado, lo mejor es aplicar herramientas preventivas como la capacidad de carga recreativa.

\subsubsection{Actividades Recreativas}

Las actividades recreativas que se pueden desarrollar en un espacio natural son muchas y variadas. Así, encontramos las de tipo lúdico o de entretenimiento, las interpretativo-educativas y/o las deportivo-aventureras. Para garantizar el confort psicológico en el transcurso de la actividad recreativa hemos de estar seguros de que el visitante está desarrollando el tipo de actividad que se ajusta a su perfil, de manera que sus expectativas se puedan ver satisfechas.

En este proceso, un elemento de confort psicológico lo constituye el espacio personal que cada visitante necesita para desarrollar la actividad y éste está directamente relacionado con el tipo de actividad, el perfil del usuario y la tipología del espacio y/o equipamiento donde se lleva a cabo la actividad. Será necesario pues realizar un estudio para conocer específicamente el Espacio Mínimo Viable para la práctica de una actividad en condiciones adecuadas. Las actividades deportivo-aventureras son las que suelen requerir mayor superficie para su práctica; para las lúdicas y de entretenimiento y las interpretativo-educativas que, en muchas ocasiones se realizan en grupo, se ha utilizado como referencia el análisis de la percepción del uso social y personal que hacen los individuos del espacio y cuyos estándares fueron propuestos en los estudios de Proxémica (Hall, 1963). Así, siguiendo estos trabajos, se ha tratado de identificar el espacio que debe haber entre un individuo y otro dependiendo de las circunstancias y el entorno. De cualquier manera, hay que pensar que diferentes culturas mantienen diferentes estándares de espacio interpersonal y que también se observan modificaciones debidas a factores sociales y culturales, de género y de preferencia individual. Otro elemento crucial es el contexto o lugar donde tiene lugar esta interacción. Teniendo en cuenta todos estos condicionantes, se puede sugerir que para garantizar el confort psicológico durante el desarrollo de una actividad interpretativa o lúdica en espacios cerrados, se debe considerar, al menos, la distancia casual-personal $(1,20$ $\mathrm{m}^{2}$ ) apuntada por Hall (1963). Se trata de una distancia aceptable en situaciones amigables con gente que incluso siendo extraños en ese momento comparten una actividad común que los une (las circunstancias y el entorno lo permiten). Para espacios abiertos, se toma la referencia de $1,50 \mathrm{~m}^{2}$ por persona para desarrollar actividades en grupo y no sentir invadida su intimidad. Para otras actividades de carácter más dinámico, se ha estimado un espacio de 4,00 $\mathrm{m}^{2}$ que, siguiendo al mismo autor, es el umbral inferior del espacio público por practicante desarrollando actividades en grupo.

Las condiciones en que se organiza la actividad son también decisivas en cuanto al confort psicológico. Así, el patrón de la visita, el nivel de frecuentación, el tamaño y tipo de los grupos son factores importantes a analizar. Los espacios naturales protegidos deben dotarse de un patrón de la visita ya que es un elemento fundamental para evitar impactos en los recursos que se desea conservar y también para facilitar el desarrollo de los programas de uso público. Un buen patrón de la visita, además de contribuir a la seguridad del visitante, aporta confort psicológico ya que permite un acceso tanto al medio físico en buenas condiciones 
(evitando la congestión y el encuentro con otros grupos, pasar dos veces por el mismo sitio, etc.), como intelectual (conocimiento de los valores patrimoniales del lugar) ya que se presentan los recursos de forma ordenada secuencial o espacialmente de forma que el visitante rápidamente puede asimilarlos sin dificultad. Todo ello contribuye de manera definitiva al confort psicológico, ya que se facilita una pauta para que el visitante no distraiga la atención en cómo evitar peligros y/o en cómo organizar y aprehender la información que se le está facilitando y pueda, por tanto, disfrutar de una experiencia sensorial añadida (sensaciones).

En cuanto a la organización de los grupos, hay que tener en cuenta: el tamaño, perfil de los componentes del grupo y los encuentros con otros grupos. En términos generales, se puede decir que cuanto más pequeño es el grupo, mayor confort se puede proveer ya que se pueden personalizar mejor las acciones, y los programas de interpretación pueden funcionar mejor (Moscardó, 1996). Existe mucha literatura científica basada en estudios empíricos en espacios naturales donde se sugiere que nunca se debe exceder un volumen de diez integrantes. También se puede comentar que cuanto más afines sean los componentes de un grupo mayor confort se constata porque todos ellos comparten motivaciones y expectativas similares y la eficiencia comunicativa va a ser mayor. Existen algunas tipologías de grupos, sobre todo los muy especializados, que son muy exigentes a este respecto y no se encuentran confortables sino es en compañía de otras personas con su mismo perfil y motivaciones, y en grupos de tamaño muy reducido; tal es el caso, por ejemplo de los ornitólogos a los que diversos autores hacen referencia en sus trabajos (Kim et al. 1997; Hvenegaard, 2002; Scott y Thigpen, 2003; Conell, 2009). Los visitantes de carácter más generalista admiten mejor las actividades en grupo y, además es más habitual, aunque desarrollan conjuntamente una actividad breve, que adquieran comportamientos grupales; en grupos más especializados suele dominar el individualismo. De esta forma, a la hora de considerar el confort psicológico de un grupo se tendrá en cuenta que, en el primer caso, necesitan un tratamiento de conjunto ya que forman un todo y la satisfacción de sus integrantes dependerá no sólo del éxito del desarrollo de la actividad recreativa sino también de las interrelaciones de dependencia de sus miembros. De estos hechos se deriva la importancia de saber componer grupos homogéneos con aspiraciones similares y de reconocer cuando hay una mala situación grupal. En cuanto al número de encuentros, los estudios de McCool (1983) establecen que encontrarse con más de 10 grupos a lo largo de una experiencia recreativa es demasiado y, por tanto, podría contribuir al disconfort psicológico del visitante.

\subsubsection{Equipamientos y Servicios}

El confort psicológico tiene también que ver con los equipamientos y, sobre todo, con los servicios ofrecidos en el espacio natural. Los equipamientos que podemos encontrar en un espacio protegido son diversos, y su planificación y desarrollo dependen de muchos factores que van desde las dimensiones del espacio, necesidades de la demanda, hasta también razones económicas; la existencia de más o menos equipamientos no es sinónimo de confort psicológico pero hay dos premisas que se deben tener en cuenta: que unos equipamientos básicos (acogida e información, aseos, señalización, aparcamientos y senderos) son necesarios incluso para acoger a los visitantes más conservacionistas; y la segunda es que los equipamientos deben estar en perfectas condiciones de mantenimiento. 
Los equipamientos de acogida e información son fundamentales para dar confianza y seguridad al visitante. La información necesaria que hay que facilitar in situ respecto al confort psicológico es fundamentalmente de tipo geográfico (localizaciones y orientación) a fin de que el visitante no se sienta nunca perdido o desorientado, y también la relativa a la seguridad y emergencias. Recomendaciones respecto al comportamiento esperado en el lugar en relación al respeto hacia los recursos patrimoniales y a otros usuarios son también muy útiles. La información que se facilite al visitante debe ser veraz y objetiva para que pueda ser procesada y evaluada por los usuarios conforme al conocimiento previo y criterios de cada uno. También es fundamental que esté convenientemente actualizada en todos los canales de información que utilice la administración del área protegida. Informaciones sesgadas, falsas o contradictorias en la información suministrada al usuario crean desconfianza hacia el lugar y los gestores.

La señalización de un espacio natural protegido es de suma importancia para crear confort psicológico en el visitante, ya que es una herramienta fundamental para proveer información, orientación y elementos para la interpretación, además de seguridad. Las señales deben estar diseñadas de forma que sean fácilmente entendibles y localizadas en lugares adecuados para que no creen desconcierto en el visitante.

Uno de los equipamientos fundamentales de las áreas protegidas son los viales o caminos por donde discurrirán los visitantes bien para trasladarse de un lugar a otro, o bien para realizar rutas interpretativas. Las campañas de observación participante llevadas a cabo nos permiten afirmar que, como regla general, a todo tipo de visitantes les resulta placentero recorrer senderos que discurran por paisajes de calidad, bien señalizados y bien mantenidos. Los visitantes de tipo generalista prefieren senderos con suelos cómodos (texturas limoarcillosas con algo de arena), no demasiado estrechos (> 1,00 m ancho), y planos o con escasa pendiente (entre 0-3\%). Los visitantes de tipo deportivo-aventurero e interpretativo-educativo admiten más dificultades en el camino si la recompensa vale la pena (paisajes de alta calidad escénica, ecosistemas sobresalientes, monumentos naturales, observación de aves raras, etc.). El diseño del trazado influye en la percepción del confort psicológico; los senderos sinuosos ofrecen una atmósfera más adecuada para visitantes que buscan la soledad ya que las curvas y la vegetación cortan las cuencas visuales e impiden la visión de otros usuarios en el mismo sendero. Para muchos usuarios en visita individual, éste es un componente fundamental del confort ya que la visión de otros usuarios en el límite del espacio público (hasta lo visible o lo audible) provoca sensación de persecución. También hay que mencionar los problemas de disconfort físico y psicológico que se registran en algunos senderos multiusuarios, cuando las relaciones entre usuarios con estilos de recreación diferente (peatones, ciclistas, jinetes a caballo, paseantes con mascotas, etc.) no son buenas. En este caso es necesario recordar que se deben aplicar estrictos códigos de etiqueta donde se especifiquen unas normas de comportamiento que faciliten el entendimiento entre todos.

Otros equipamientos deseables para facilitar el confort psicológico son los interpretativoeducativos ya que están en la base del acceso intelectual al patrimonio. Los más representativos son las rutas interpretativas y los centros de interpretación que no siempre existen en los espacios naturales protegidos. Según las encuestas realizadas, este tipo de equipamientos siempre resulta muy bien valorado por los visitantes.

Por su parte, los servicios son el conjunto de actividades, acciones y programas necesarios para la práctica de actividades recreativas; su desarrollo suele requerir el apoyo de equi- 
pamientos y/o personal especializado. El confort psicológico y los servicios presentan una relación difícil de establecer porque, es muy variable dependiendo del perfil del visitante y de la calidad del servicio ofrecido, entendiendo ésta como el cumplimiento de la expectativa o la necesidad del visitante. El público más generalista necesita habitualmente más equipamientos y servicios; mientras que los visitantes especializados suelen obviarlos, e incluso pueden llegar a valorar negativamente su sobredimensionamiento.

Existe toda una serie de servicios básicos que todo espacio natural debe contemplar como son los de limpieza y mantenimiento, gestión de residuos, primeros auxilios y seguridad además de los informativos. Otros servicios adicionales en espacios protegidos con una gestión más sofisticada son aquellos pensados especialmente para públicos con alguna discapacidad física o psíquica. Los servicios interpretativos deberían ser un elemento irrenunciable que merece el público de cualquier espacio protegido y que si se presta en condiciones apropiadas y con guías-intérpretes cualificados garantiza el acceso intelectual al patrimonio del lugar. De ahí, el enorme valor que tienen los recursos humanos en el desempeño de los programas de información e interpretación, y en la conducción y guiaje de grupos; se podría decir que son el mejor vector del confort psicológico.

\subsection{Factores relacionados con los visitantes}

El confort psicológico de los visitantes tiene que ver en gran medida con complicados procesos mentales propios de las personas; por ello se puede decir que son los más difíciles de abordar, debido a su carácter en parte subjetivo y cambiante según las circunstancias. Para poder analizar de una forma coherente cómo se configura el confort psicológico, se ha desagregado el proceso en la secuencia que aparece en la figura 1.

Figura 1

SECUENCIA DE LA CONFIGURACIÓN DEL CONFORT PSICOLÓGICO DE LOS VISITANTES

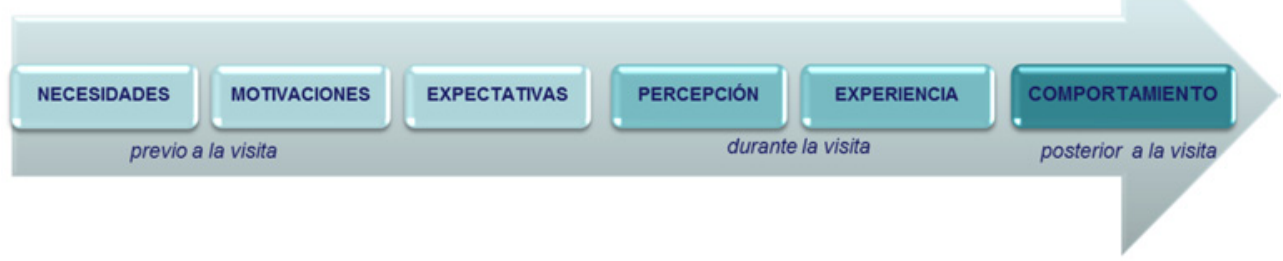

Necesidades, motivaciones y expectativas son procesos que se forjan con antelación a la vista al espacio natural y dependen, en parte, de los rasgos de personalidad del individuo. La percepción y experiencia recreativa tienen lugar in situ y es responsabilidad de los gestores de los espacios el que estos procesos se produzcan con las máximas garantías de confort físico y psicológico. El comportamiento se comienza a producir durante la visita pero se pretende que pase a formar parte del estilo de vida del visitante en el futuro.

Las necesidades de recreación o entretenimiento afloran cuando las de carácter básico han sido ya satisfechas. La recreación incluye toda una serie de actividades que el individuo 
lleva a cabo durante su tiempo libre y que satisfacen la necesidad de relajación física y mental. Esta necesidad como es obvio es muy variable dependiendo de las personas, de su estilo de vida, etc. Las necesidades recreativas basadas en la visita a espacios naturales tienen que ver con vínculos muy primarios que unen a las personas con la naturaleza, lo que se conoce bajo el concepto de biofilia (Wilson, 1986).

Las motivaciones son las fuerzas internas que conducen a una persona a actuar para satisfacer unas necesidades, en este caso las recreativas. Es evidente que éstas varían según los rasgos de personalidad del visitante $\mathrm{y}$, desde esta perspectiva, conviene recordar el trabajo anteriormente citado de Plog. Independientemente de las relaciones existentes entre las motivaciones y los rasgos de personalidad del visitante, existen numerosas propuestas de clasificación de las motivaciones. Así, Luo y Deng (2008) y, posteriormente, Line y Costen (2011) han trabajado en un sistema de motivaciones de los visitantes de espacios naturales que incluye básicamente cuatro tipos que, en nuestra opinión, resumen bien las propuestas en la literatura científica. De esta forma, las motivaciones que llevan a una persona a visitar un espacio natural protegido pueden ser: una nueva vía de desarrollo personal, vuelta a la naturaleza, conocimiento y salud, y escapada. Además, estos autores encuentran coincidencias positivas en las relaciones entre actitudes medioambientales de los visitantes y comportamiento durante la visita a espacios protegidos.

El conocimiento de las necesidades y motivaciones de los visitantes se lleva a cabo mediante encuestas especiales donde se realizan preguntas acerca del nivel de conocimiento del sitio, rasgos de personalidad, experiencias previas y también a través de cuestiones sobre los motivos de la visita. Estas encuestas son más complejas de abordar que las de perfil sociodemográfico porque el encuestado no suele estar acostumbrado a contestar sobre aspectos de su persona que le resultan incluso complejos de expresar. Se suelen utilizar cuestionarios con respuestas múltiples para que pueda elegir, de forma que la entrevista pueda realizarse de una forma cómoda y ágil. No obstante, también se suelen dejar cuestiones abiertas donde pueden opinar y, de esta forma, afloran temas interesantes que no han podido ser recogidos en las preguntas.

Necesidades y motivaciones son el terreno abonado de donde surgen las expectativas, pero para ello ha de agregarse un nuevo elemento que es la imagen del destino o imagen turística. Nuestra mente construye las expectativas a partir de la información que recibe sobre el lugar y crea una imagen proyectada. Echtner y Ritchie (1991) apuntaban que los individuos pueden tener una imagen de un destino incluso sin haberlo visitado o haber sido expuestos a fuentes de información comerciales, ya que las personas a lo largo de su vida van acumulando información relacionada con factores históricos, políticos, económicos y sociales que van conformando su imagen sobre el destino en cuestión. La información que recibe un potencial visitante puede proceder de diversas fuentes a las que Gartner (1993) denomina agentes de formación de la imagen y Baloglu y McCleary (1999) llaman factores estímulo, y que son: las oficiales (en nuestro caso la administración del espacio protegido), comerciales (que serían los proveedores de servicios turísticos en la zona), y de otros prescriptores. Sobre la primera fuente hay que resaltar, como ya se ha mencionado, la importancia de la veracidad de la información suministrada ya que una información errónea o sin actualizar puede frustrar las expectativas de un visitante en cuanto llegue al lugar y configure la imagen percibida, creando un gran disconfort psicológico difícil de restaurar posteriormente aunque existan otras oportunidades en el lugar. La información proveniente de fuentes comerciales suele ser 
interesada y, en muchas ocasiones no da la confianza suficiente al usuario. Los prescriptores son otros usuarios que han visitado previamente el lugar; dan incluso una confianza mayor que la proporcionada por las fuentes oficiales. Así, además del boca-oreja, hay que destacar en la difusión de esta información los programas televisivos con el nuevo formato tipo Callejeros Viajeros, Españoles en el mundo, etc., pero sobre todo, sobresale la contribución de las redes sociales (blogs, facebook, twitter, etc.). Parte del éxito y la confianza que inspiran estos medios es debido, en primer lugar, a que son interactivos; la información fluye rápidamente y en los dos sentidos, y, además, se debe también a que los mensajes que se transmiten suelen tener una componente emocional que no suele contener la información oficial; es decir, en estos medios se suele hablar de las sensaciones, emociones y sentimientos, en definitiva de la experiencia vivida, más que de datos objetivos sobre el lugar. Estas componentes emocionales sirven para alimentar las motivaciones y expectativas de otros visitantes que es lo que necesitan en esa fase del proceso.

Ya en el espacio natural, se produce la percepción que es un proceso mediante el cual un individuo recibe información del entorno a través de los sentidos, la selecciona, la organiza y la interpreta para crearse una imagen significativa o percibida (Mayo y Jarvis, 1981). Al ser un proceso que se realiza ya in situ y, para poder dar significado a la información que el individuo recibe, es necesaria una integración de la misma con elementos cognitivos previos (recuerdos, ideas, creencias, sentimientos previos, modelos, experiencias anteriores, etc.). Por tanto, vemos que en este proceso interviene la memoria, el aprendizaje y la inteligencia tal como apuntaba Feldman (1999) cuando destacaba que esta actividad no sólo era tarea de los órganos sensoriales sino también del cerebro. Si en alguna fase del proceso es importante garantizar un confort psicológico al visitante es en ésta, ya que en ese momento se produce la última oportunidad de poder influir en la impresión personal que el visitante se va a forjar del lugar. Por tanto, éste debe estar centrado en absorber toda la información que el entorno le facilita para poder construir la percepción que se persigue; la administración del sitio en su programa de interpretación debe haber identificado la imagen y mensajes del lugar que quiere transmitir, procurando que nada ni nadie interfiera en este proceso y teniendo en cuenta que la percepción puede variar según los rasgos de personalidad, conocimientos previos, experiencia del observador y desarrollo cultural de cada individuo tal como apuntaban Pizam y Mansfel (1999) y Shiffman y Lazar (2000).

Un elemento importante a considerar en la percepción apuntado por Pike y Ryan (2005) y Andrade (2012) entre otros, es además de la dimensión cognitiva o racional (conocimiento de los atributos: recursos, atractivos, equipamientos y servicios, etc.), la afectiva o emocional. La información cognitiva admite pocas posibilidades de equívoco porque los recursos o atractivos son los que existen, aunque es evidente que deben presentarse al público adecuadamente y en las mejores condiciones posibles de conservación. La información emocional es subjetiva y puede ser estimulada o mejorada.

Derivado del análisis de la puesta en marcha de diversos programas de interpretación y centros de visitantes, este equipo de investigación ha identificado técnicas para la mejora de la percepción de un lugar. En primer lugar, hay que comentar que el espacio debe estar bien conservado, lo más próximo a la integridad ecológica y paisajística que sea posible y ningún elemento extraño debe empañar la belleza escénica. Por otra parte, hay que conseguir un impacto sensorial mediante la utilización de todos los sentidos en el acto de aprehender la 
información. Como ya se ha comentado, conocer los rasgos de personalidad de la audiencia es fundamental; en espacios naturales con recursos difíciles de interpretar para el público generalista hay que identificar iconos interpretativos que les resulten más familiares, amigables y fáciles de aprehender. Es también muy importante utilizar el patrimonio intangible, ya que está muy ligado a las personas y, por tanto, a las emociones.

A partir del momento en que se comienza a forjar la percepción, otros procesos internos que configuran la experiencia recreativa, se ponen en marcha. Las experiencias recreativas son el resultado de vivir determinadas situaciones, por tanto se establece una conexión muy directa entre el lugar y el visitante. Durante la experiencia se produce un aprendizaje de cosas nuevas debido a la información cognitiva que ha llegado al cerebro, y esto puede considerarse ya como una acción positiva; pero también e igualmente importante se pueden desencadenar toda una serie de emociones como hemos comentado. Así pues, se puede decir que las experiencias aportan a los visitantes valores sensoriales, emocionales, cognitivos, conductistas y de relación.

Hay que comentar que hasta fechas recientes, incluso en muchos sitios todavía, los programas de interpretación o la información facilitada a un visitante en un espacio protegido se queda en el campo del conocimiento cognitivo. Cada vez con más fuerza, se apunta hacia la consecución de emociones; se habla de «tener una experiencia» incluso se extiende rápidamente el concepto de «Turismo experiencial» haciendo referencia a la componente emocional que debe comportar la visita a un lugar. Y parece que esto no va a ser una moda pasajera sino que es una clara tendencia de futuro. Se debe aspirar en definitiva, a que las emociones formen parte de los atributos del lugar (emociones como un fin en sí mismo). En este sentido, cabe destacar algunos ejemplos de campañas publicitarias en España que basan la diferenciación del destino en estos aspectos sensitivo-emocionales como es el caso de la «Marca Extremadura 2010», «Galicia ¿Me guardas un secreto?» o los de TourEspaña «I need Spain».

El objetivo del gestor del espacio natural tiene que ser pues que el visitante pueda experimentar emociones que le lleven a generar sentimientos positivos de satisfacción que, a su vez, den lugar a actitudes de aprecio hacia la conservación y el disfrute del espacio natural. Para conseguir esto, es necesario que el visitante se encuentre en estado de confort psicológico. Hay que añadir además que, para reforzar este proceso, los programas de interpretación deben recurrir al apoyo de guías-intérpretes, por varias razones. En primer lugar, porque muchos visitantes no son capaces por sí solos de conectar en toda su dimensión con los estímulos que envía la naturaleza y un guía-interprete puede ayudar a aprehender estas sensaciones; en segundo lugar porque los seres vivos y, en particular los humanos, son los más capaces de generar emociones a otro ser humano. Se deriva obviamente de estas declaraciones, la importancia que tiene la formación del personal encargado de esta responsabilidad. En muchas ocasiones, el éxito de la visita está en sus manos y pueden ser capaces de resolver situaciones aún en condiciones externas de confort adversas.

La experiencia recreativa es sometida por nuestra mente a una evaluación que se traduce en un juicio sobre si el rendimiento es: exactamente como se esperaba, mejor de lo que se esperaba, o peor de lo que se esperaba. En los dos primeros casos se producirá satisfacción, mientras que en el tercer caso se constatará claramente una insatisfacción. La satisfacción es un sentimiento de bienestar o placer que se tiene cuando se ha colmado un deseo o cubierto una necesidad. Este sentimiento es producto de la valoración cognitiva (Hunt 1975; Day, 1984; Fornell, 1992; Engel et al., 1993), y/o emocional (Swan y Oliver, 1989; Oliver, 1993; 
Halstead et al., 1994; Spreng et al., 1996; Baker y Cromptom, 2000) que se deriva de la experiencia en su visita a un espacio protegido. Se podría decir que la satisfacción es la expresión máxima del confort psicológico durante el proceso de la experiencia recreativa de un visitante.

Los primeros elementos que entran a formar parte de la evaluación de la experiencia son los atributos o atractivos del lugar, por eso numerosos autores hacen hincapié en la importancia de hacer coincidir la imagen proyectada del lugar con la que perciben los visitantes (Kotler et al., 1993). Caso que de esta primera evaluación se derivara un sentimiento de insatisfacción, una situación de disconfort psicológico se apoderaría del visitante de manera que un estado de ánimo pesimista y una actitud negativa se instaurarían en las subsecuentes valoraciones de los atributos del lugar, pudiendo este estado mental perturbar el criterio e impedir que se disfruten otros elementos del lugar que no merecerían una valoración negativa. Hay que procurar que no se frustren las expectativas de los visitantes ya que como Hsu et al., (2010) y Devesa et al. (2010) han demostrado existen poderosos vínculos entre las motivaciones y expectativas de los turistas y sus actitudes y comportamientos durante la visita de los destinos.

Autores como Tian-Cole y Crompton (2003) proponen considerar, además de la satisfacción global de la visita, la calidad de la experiencia. Ésta hace referencia a los beneficios obtenidos en el lugar, considerando como tales la calidad de los equipamientos y servicios y la atención al público del personal (amabilidad, educación, preparación, profesionalidad, etc.), ya que estos elementos son susceptibles de ser gestionados.

Adquirir información sobre la satisfacción global de los visitantes se puede abordar a partir de encuestas de satisfacción. Muchas administraciones de áreas protegidas suelen llevar a cabo regularmente este tipo de encuestas como el Servicio de Parques Nacionales de Estados Unidos (USDA Forest Service, 2012) que incluye preguntas directas sobre el grado de satisfacción obtenido con la visita y algunas específicas sobre el trato recibido de los empleados de los parques, sobre los equipamientos, etc. La satisfacción también se puede evaluar a partir de la observación directa y la observación participante.

La reacción ligada al sentimiento de satisfacción, además de un estado de ánimo positivo, es una actitud y un comportamiento. Determinados comportamientos son el fin último que persigue un gestor de un área protegida y son aquellos que pueden contribuir a la conservación del patrimonio y los recursos del lugar. No obstante, se puede ir más allá y plantearse objetivos más ambiciosos en relación a actitudes y comportamientos que puedan transcender al propio momento de la visita e influir en la vida cotidiana del visitante. Hay que mencionar que los sentimientos son más poderosos cuando se producen durante el momento de la visita, pero que pueden persistir en ausencia de los estímulos externos que los generaron, de ahí su valor para potenciar conductas posteriores en el visitante a medio y largo plazo.

Al ser el comportamiento un resultado del proceso, resulta difícil influir desde el punto de vista del confort psicológico porque el visitante está prácticamente acabando su visita y porque ex situ va a ser más complicado mantener los vínculos con el espacio natural salvo que así lo desee. Actualmente hay muchas posibilidades de seguir en contacto con los visitantes a través de las nuevas tecnologías si el espacio natural mantiene un sitio web convenientemente actualizado y con herramientas interactivas que permitan reforzar los mensajes y mantener vivos los sentimientos y los recuerdos. 
A tenor de lo comentado, hay que resaltar que el comportamiento del visitante in situ es una excelente herramienta para valorar la satisfacción y el nivel de consecución de los objetivos planteados en los programas de interpretación y sensibilización. Por tanto, la observación directa de determinadas actitudes y comportamientos y la observación participante nos sirve además de como evaluación del confort psicológico del visitante, como indicador de la actividad gestora. De esta forma, investigaciones recientes (Faullant et al., 2008; Wang et al., 2009; Zabkar et al., 2010; Yuksel et al., 2010; Rodger et al., 2012) han establecido que la satisfacción de los visitantes es un factor determinante de la fidelización al lugar y la recomendación del sitio a otros visitantes. Por ello, basándonos en estos trabajos, hay que pensar además que si un visitante ha podido completar el proceso de forma satisfactoria es que lo ha hecho en condiciones de confort psicológico y al final está actuando como prescriptor del sitio.

\section{RESULTADOS DEL ANÁLISIS DE LOS CASOS DE ESTUDIO}

Desde la perspectiva planteada en los apartados anteriores, se han analizado cuatro casos de estudio de diversos espacios protegidos de la Comunidad Valenciana (fig. 2) que se encuentran dentro de la Red Natura 2000 (Directiva Europea Habitats), además de detentar otras figuras de protección internacional.

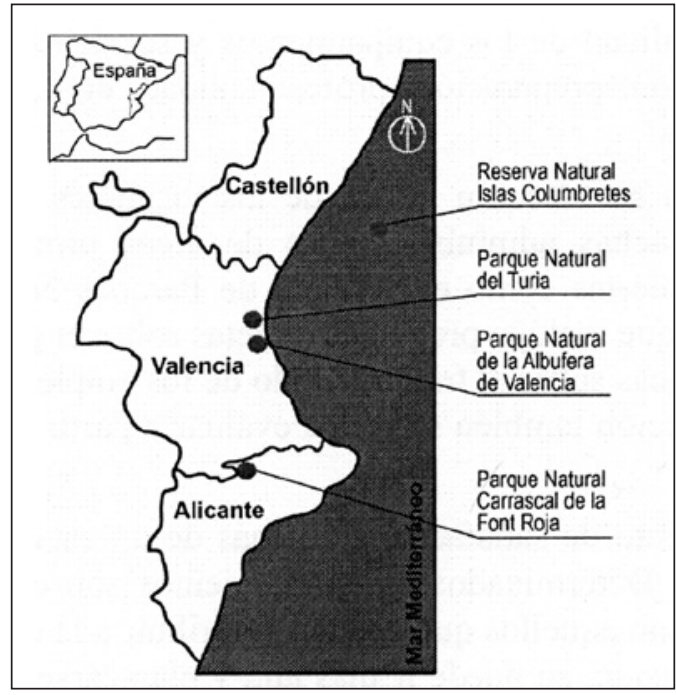

El Parque Natural del Carrascal de la Font Roja es un ecosistema característico de la montaña media mediterránea. Tiene una superficie de 2.450 ha, abarcando las dos vertientes de la sierra del Menejador (1.352 m). Las Islas Columbretes constituyen un archipiélago en el Mar Mediterráneo, formado por un conjunto de pequeñas islas y escollos volcánicos 
situados a $56 \mathrm{~km}$ del cabo de Oropesa (Castellón). Tienen en total una extensión emergida de 19 ha, declarada como Reserva Natural, y una Reserva Marina de 4.400 ha de superficie acuática alrededor del área emergida. L’Albufera de Valencia constituye uno de los mayores humedales de la Península Ibérica. Se encuentra en la costa mediterránea y fue el primer Parque Natural que se declaró de la Comunidad Valenciana (1986) e incluye 21.000 ha. El Parque Natural del Turia abarca una superficie de 4.692 ha y está constituido básicamente por un corredor verde de bosque de ribera instalado en la llanura del río Turia a su paso por las comarcas de Los Serranos, El Camp de Túria y L'Horta, llegando hasta la ciudad de Valencia. También incluye masas boscosas de pinares y matorral mediterráneos que constituyen los auténticos pulmones verdes del área metropolitana.

En relación a los elementos de confort físico, hay que destacar que en estos casos de estudio el clima ha jugado siempre a favor de los visitantes ya que nos encontrarnos en el ámbito del clima mediterráneo, con un gran número de días de insolación, pocas lluvias diurnas y, en general escasos riesgos climáticos. En el caso de la Font Roja, durante la estación invernal pueden tener lugar ventiscas y nevadas, constatándose temperaturas en algunos días de estos meses por debajo de $0^{\circ} \mathrm{C}$, por lo que se produce una falta de confort térmico pese al atractivo que supone siempre la presencia de nieve en las montañas de estas latitudes. El resto del año, las temperaturas están dentro de los rangos considerados como confortables para el desarrollo de actividades al aire libre. En el caso de las Islas Columbretes, el factor de disconfort climático existente tiene que ver básicamente con el viento ya que puede dificultar la travesía y el abordaje a tierra. Estas situaciones se producen fundamentalmente en invierno por lo que los servicios de uso público se cancelan durante este periodo. Así, las visitas se llevan a cabo en verano, aunque durante esta estación se registran días de altas temperaturas $\left(>35^{\circ} \mathrm{C}\right)$ que combinadas con una alta humedad relativa $(>60 \%)$ dan lugar a situaciones de elevado disconfort termo-higrométrico para el cuerpo humano. El único riesgo climático importante en l'Albufera de Valencia y en el Parque Natural del Turia viene dado por las lluvias de fuerte intensidad horaria en otoño que hacen impracticable la visita durante esos días y, en el caso particular del río Turia podría entrañar riesgo de inundación de ciertas áreas de uso público. Al igual que en las Islas Columbretes, se registran días en verano de elevado disconfort termo-higrométrico tan sólo compensado por la presencia de brisas marinas en el caso de l'Albufera.

Por lo que respecta a la seguridad que presentan los espacios estudiados, hay que decir que en general, es buena y por tanto, éste no es un factor que decisivamente afecte al confort físico ni psicológico de los visitantes. En el caso de la Font Roja y del Turia, hay que comentar que los aspectos relacionados con los incendios están bien contemplados; existen Programas de Vigilancia y un Plan Integral de Prevención de Incendios. No obstante, habría que tomar medidas más estrictas de información y prevención para los días de riesgo meteorológico por nevadas o lluvias de fuerte intensidad horaria. El Turia cuenta además con un Plan de Emergencias y Evacuación con 25 puntos de acceso de emergencia ya que debido a la elevada frecuentación que se registra es necesario para poder garantizar la seguridad de los usuarios del parque. En el caso de l'Albufera, la zona de uso intensivo del Racó de 1’Olla no cuenta con un Plan de Seguridad propio, se aplica el plan genérico para toda la zona cercana de El Saler, en el que se establecen actuaciones dirigidas a la disminución del riesgo de incendio. Hay que mencionar que el centro de interpretación 
necesita algunas adecuaciones para cumplir con los requisitos de seguridad necesarios para el desarrollo de actividades de uso público. Garantizar el confort físico en las Islas Columbretes es más complejo, debido su lejanía de tierra firme. Uno de los principales problemas tiene que ver con la zona de desembarco de Puerto Tofiño, que puede presentar problemas, sobre todo en los días donde hay marejada. Hay que hacer también mención que, en caso de producirse accidentes dentro de l'Illa Grossa, que es la mayor del archipiélago donde están instalados todos los equipamientos de uso público, las unidades de socorro más próximas se encuentran en Castellón y la evacuación debe hacerse en helicóptero o en alguna embarcación rápida.

Los espacios analizados en este trabajo se incluyen en las categorías de abiertos, abiertos con barreras físicas y cerrados. Los abiertos son lagunas, bosques, islas y riberas fluviales. Entre los abiertos con barreras físicas incluimos: senderos, torres-mirador, áreas de pic-nic y aparcamientos; los espacios cerrados son los centros de información/interpretación y los observatorios. En cuanto a los espacios al aire libre analizados, hay que mencionar que tanto la Font Roja como el Turia y l'Albufera presentan espacios llanos o de pendientes suaves y con arbolado no excesivamente denso, por lo que permiten buenas cuencas visuales en el plano medio. Agradables paisajes sonoros (movimientos de ramas, aves, agua discurriendo, etc.) completan estos escenarios por lo que se podría decir que resultan paisajes relajantes y confortables para la mayoría del público generalista. Posiblemente al público especializado le gustaría una mayor densidad de la vegetación más cercana al bosque prístino original. Por lo que respecta a las Islas Columbretes, señalar que se trata de un espacio muy abierto y elevado sobre el horizonte que permite una visión de $360^{\circ}$ sobre el mar Mediterráneo. Las sensaciones que causa en el visitante pueden ser contradictorias; así, desde aquellos que lo disfrutan como un espacio especial alejado de lo urbano y donde los únicos sonidos provienen de las aves, del mar y del viento, hasta otros visitantes que pueden desatar sensaciones de agorafobia o de aislamiento con la consecuente inseguridad psicológica que llevan aparejadas.

Por otra parte y, en relación con la zonificación de los espacios, se han analizado preferentemente las zonas de uso intensivo: Ermita dels Lliris en la Font Roja; l'Illa Grossa del archipiélago de Columbretes, el Racó de l'Olla en l'Albufera y el sendero multiusuarios y áreas recreativas de Manises y Riba-roja de Túria en el Parque Natural del Turia. Los senderos han sido también objeto de estudio especial.

En relación a la frecuentación, hay que decir que es un tema complejo de abordar por las dificultades intrínsecas que conlleva controlar la afluencia de público a estos espacios abiertos. No hay demasiadas fuentes informativas que faciliten datos y, en ocasiones son contradictorios. En este trabajo se ha utilizado el informe Eroski-Consumer (2012) que realiza anualmente a partir de datos proporcionados por la comunidad autónoma referidos exclusivamente a visitantes que acceden a los centros de visitantes, por lo que se puede decir sin dudar que el número de visitas es realmente mucho mayor. Hay que comentar que del Parque Natural del Turia no se poseen datos oficiales sino que han sido estimaciones realizadas por el personal técnico de este espacio protegido. No obstante todo, podemos tomar como referencia, al menos, la magnitud del volumen registrado para cada espacio. A este respecto, hay que decir que desde que se inició el estudio hasta 2011, que es el último año del que se disponen datos, el volumen de visitantes ha ido creciendo. Las últimas esta- 
dísticas nos informan de que la Font Roja recibió 59.797 visitantes, l'Albufera 38.790 y las Islas Columbretes 19.625, siendo este último espacio protegido el que ha experimentado un crecimiento mayor en los últimos años. Las estimaciones realizadas para el Turia arrojan la cifra de 750.000 visitantes, corroborada por conteos puntuales realizados por la Universitat Politècnica de Valencia y la Universitat d'Alacant entre 2009 y 2012.

Una primera impresión de estos datos en relación con la superficie de los parques nos informa que el más saturado es el Parque Natural del Turia, seguido de la Font Roja. Con anterioridad a este trabajo, se estudió la capacidad de carga recreativa de estos espacios y de l'Albufera (Viñals et al. 2004; Morant y Viñals, 2010), y se puede decir que en términos generales estaban dentro de los márgenes razonables de utilización. Se han registrado problemas puntuales en el sendero multiusuarios del río Turia que presenta congestión de tráfico ciclista en ciertas franjas horarias los fines de semana y festivos. Las Islas Columbretes también presentan congestión en algunos días de verano debido a la llegada inesperada de visitantes en yates privados, sin haber concertado previamente. Otra situación de saturación se presenta con ocasión de eventos especiales como la Romería de la Virgen del Lliris en la Font Roja. En las encuestas realizadas en estos parques, se preguntaba acerca de la percepción de la congestión y, en el caso del Turia que es el que registra este problema con mayor intensidad, el 51,2\% de los visitantes respondía que sí la percibía pero esta circunstancia no afectaba a su confort psicológico y no les pesaba demasiado en su valoración del lugar. Se puede comentar pues que la gestión de los visitantes es mejorable en todos los parques y se podrían incluso minimizar los impactos sobre los recursos, aplicando medidas de gestión de la capacidad de carga recreativa. Ello mejoraría la percepción del espacio por parte del visitante y por tanto, contribuiría a incrementar su confort psicológico.

Respecto a las actividades recreativas, hay que señalar que las más practicadas en la Font Roja, 1'Albufera e Islas Columbretes han sido las interpretativo-educativas concertadas guiadas y las lúdicas o de entretenimiento espontáneas (salvo en el caso las Islas Columbretes que hay que concertar siempre la visita), representadas básicamente por paseos al aire libre. El Parque Natural del Turia es un caso un poco especial y las actividades más habituales son las lúdicas o de entretenimiento y las deportivo-aventureras llevadas a cabo de forma individual o en grupos auto-organizados. En este parque, pasear es la actividad más popular con un $70.6 \%$ de las respuestas debido a que los usuarios viven muy cerca del corredor verde. Esta actividad es la más habitual registrada en bosques cerca de las ciudades tal como apuntan Lindhagen (1996), Guyer y Polland (1997) y Roovers et al. (2002). Este grupo de usuarios incluye a los corredores (joggers) y los paseantes con mascotas; también es reseñable la presencia de visitantes en las áreas recreativas de Manises y Riba-roja de Túria llevando a cabo actividades lúdicas (pic-nic, etc.) durante los fines de semana. El siguiente grupo y experimentando el mayor crecimiento en la tendencia son los ciclistas con un 64.2\%. Las razones de la alta presencia de ciclistas expuestas en las respuestas de los cuestionarios están en relación con la adecuación del sendero para la práctica de esta actividad. También se practica en este espacio protegido la equitación recreativa, existiendo desde 2013 un Itinerario Ecuestre homologado (IE-031) por la Real Federación Hípica Española de 46,20 km de recorrido. Los jinetes a caballo representan el $3,3 \%$, siendo un porcentaje que se ha mantenido estable durante estos años. Los visitantes del Turia frecuentan este lugar con una periodicidad de entre 2 y 4 veces por semana 
$(52,5 \%)$, y se caracterizan por residir en los municipios de las inmediaciones. Se podría decir, en definitiva, que este parque actúa como espacio de «Recreación de Proximidad» como ya apuntaban Schipperijn et al. (2010) en otros casos de estudio en relación a este tipo de parques periurbanos.

En cuanto a equipamientos y servicios, hay que señalar que el Turia y l'Albufera son los parques que presentan mejores accesos desde el exterior, mientras que las Islas Columbretes tiene las mayores limitaciones, ya que solo se puede desembarcar en l'Illa Grossa en el embarcadero de Puerto Tofiño donde únicamente lanchas zodiac y barcos muy pequeños pueden atracar. Equipamientos básicos como son los aseos, existen en la Font Roja y en l'Albufera pero no así en las Islas Columbretes debido a la inexistencia de un sistema de tratamiento de aguas residuales en la isla. Esta situación, según se ha constatado crea una situación de disconfort entre los visitantes que se ven obligados a embarcar para poder hacer uso de este equipamiento básico. Tampoco en el Turia están habitualmente disponibles los aseos públicos.

Todos los parques presentan un número suficiente de senderos señalizados no motorizados y seguros y, salvo en el caso del Turia que permite diversos tipos de usuarios (peatones, paseantes con mascotas, ciclistas y jinetes a caballo), el resto están restringidos a uso peatonal. Estos senderos, no obstante, están concebidos más como viales de paso, que como senderos interpretativos, además no son circulares en muchas ocasiones, sino que el mismo vial es utilizado en los dos sentidos de la visita, lo que resta expectación. Este diseño lineal del patrón de la visita, en algunos casos está justificado por imperativos de la geomorfología existente como el caso del corredor verde del Turia o en las Islas Columbretes donde la geografía agreste no facilita el tránsito, pero en el resto de parques se podría incrementar el confort si se cambiara.

La señalización de todos los parques, salvo en parte el Turia, adolece de una clara intencionalidad, siendo insuficientes las señales direccionales, e incompletos los contenidos de las informativas y las interpretativas cuando existen. La falta de información provoca en visitantes individuales sensaciones de disconfort psicológico e inseguridad. El Parque Natural del Turia ha sido el más recientemente señalizado y ha paliado parcialmente estas deficiencias, gracias a la instalación de señales informativas, direccionales y de emergencia a lo largo del sendero multiusuario.

Es remarcable que todos los parques cuentan con centro de información/interpretación. En los casos de l'Albufera y la Font Roja se trata de antiguos edificios rehabilitados para tal fin con los inconvenientes que esto lleva siempre consigo. Así pues, se trata de construcciones no específicamente aptas para el fin establecido o sobredimensionadas como es en el caso de la Font Roja. El caso de las Islas Columbretes está instalado en el faro. El centro de interpretación del Parque Natural del Turia en una casa de madera junto al cauce del río en el término de Vilamarxant. Respecto a los servicios informativos e interpretativos que son los que contribuyen en mayor medida a satisfacer las expectativas cognitivas de los visitantes, hay que reseñar que existe al alcance de los visitantes previamente a su visita, información disponible en la página web de la Conselleria de Medio Ambiente, Agua, Urbanismo y Vivienda de la Generalidad Valenciana. En los propios parques existen folletos y, en ocasiones paneles informativos con información veraz, lo que proporciona una imagen proyectada bastante adecuada a la realidad de cada parque, lo cual contribuye a 
poder crear unas expectativas con posibilidades reales de verse satisfechas durante la visita (imagen percibida). Las exposiciones interpretativas en los centros son antiguas y mejorables en todos los casos y, en relación a los servicios interpretativos, hay que comentar que el personal dedicado a este servicio (guías-intérpretes y monitores) ha ido en regresión durante los últimos años con el consiguiente perjuicio para los visitantes. Por ello, se puede decir que en estos momentos la componente emocional de la visita está descuidada.

A partir de estudios detallados de Morant (2007), se tienen datos de los visitantes de la Font Roja, Albufera e islas Columbretes y se puede decir que presentan similitudes en cuanto al perfil socio-demográfico y rasgos de personalidad. Se podrían clasificar perfectamente como visitantes generalistas con una capacidad perceptual media-alta; es decir, no son demasiado exigentes en cuanto a requerimientos de espacio para llevar a cabo las actividades recreativas y toleran bien la presencia de otras personas mientras no se llegue a la saturación. El tipo de visita más habitual es la concertada: escolares en días laborables y familias durante los fines de semana. En las Islas Columbretes, existe un cupo de 2-3 grupos concertados de 20 personas al día, si bien es bastante complejo controlar los desembarcos sin autorización como se ha comentado.

Del Parque natural del Turia, se sabe a partir de Viñals et al. (2011) que el visitante-tipo muestra ciertos rasgos de especialización hacia actividades deportivas y de aventura. Así, se podría definir como: hombre $(63,3 \%)$, entre 36 y 50 años de edad $(36,9 \%)$, que trabaja en el sector servicios $(46 \%, 0 \%)$ y con un nivel de estudios alto $(36,1 \%)$. La mayor parte de los visitantes se concentran en los fines de semana o periodos vacacionales. Corredores y ciclistas presentan unos rasgos de personalidad peculiares (lo que la literatura turística anglosajona denomina «more-more»), caracterizados por una notable vigorexia tal como la describe Pope et al. (2001). Son, según Corbett (2006), generalmente hombres que viven en las ciudades y desarrollan trabajos poco dinámicos y cuando disponen de tiempo libre lo dedican a hacer actividades que implican un gran esfuerzo físico en espacios abiertos.

Entre las motivaciones que estimulan la visita de estos espacios protegidos, a tenor de las respuestas y comentarios libres que se han podido analizar en los cuestionarios, destacan la «escapada» y «contacto con la naturaleza». En el caso de los visitantes del Turia se podría resaltar también secundariamente el desarrollo personal como motivación (espíritu de superación, fijarse metas físicas, etc.). En l'Albufera y la Font Roja el afán de conocimiento parece que es la motivación de los grupos concertados.

Además, derivado de las encuestas realizadas por los gestores de los parques y por los trabajos realizados por este equipo, se observa que las expectativas del público generalista y también de los especialistas han sido satisfechas tras la visita. Este mérito, hay que atribuírselo, en gran medida, a los propios espacios que detentan una gran belleza y unos recursos muy atractivos y únicos, también hay que mencionar en este sentido que la imagen proyectada por la información ofrecida previa a la visita y la percibida se aproximaban; incluso tras el trabajo realizado de observación participante podríamos decir que la imagen percibida fue mejor de lo que se esperaba. Los encuestados mostraron un grado medio-alto de satisfacción con la experiencia en un $84,10 \%$ de los casos en la Font Roja; un $70.63 \%$ en las Islas Columbretes y un 76,9\% de los visitantes del Turia. De estos resultados podemos deducir la intención de volver a visitar el parque y de recomendárselo a otras personas. 


\section{CONCLUSIONES}

A la vista de lo expuesto, se pone de manifiesto la necesidad de prestar especial atención a los factores que condicionan el confort psicológico ya que están íntimamente vinculados a la satisfacción de la experiencia recreativa.

Así, no solo es muy importante que los espacios naturales protegidos estén perfectamente conservados y gestionados desde el punto de vista patrimonial, sino que la administración de estos lugares debe ir más allá y preocuparse porque los visitantes tengan un acceso físico e intelectual en condiciones, que permita que tengan una experiencia de calidad y satisfactoria en el lugar y, si es posible, que desarrollen emociones y sentimientos positivos que les lleven hacia actitudes y comportamientos cada vez más comprometidos con la conservación y protección de los mismos.

El confort psicológico no se puede entender como un elemento estándar aplicable a todos los perfiles de visitantes sino que varía mucho con las características sociodemográficas y rasgos de personalidad de cada individuo, así como con las expectativas en torno a la experiencia recreativa a realizar. Los gestores de los espacios protegidos deben proveer de confort psicológico a los visitantes antes incluso de la visita, cuando se está forjando la imagen proyectada ya que es determinante para la generación de las expectativas sobre el lugar. No obstante, será in situ cuando se deben realizar todos los esfuerzos para que la experiencia sea satisfactoria y así se consigan la actitud y comportamientos esperados de los visitantes. El confort psicológico debe ser un concepto que se asocie con la calidad de la experiencia.

Respecto a la satisfacción de la experiencia recreativa en los casos de estudio analizados, señalar que es el público generalista es el más fácil de satisfacer en cuanto al confort psicológico. Además de los visitantes que desarrollan actividades lúdicas y de entretenimiento, se incluyen también los visitantes que acuden en grupos organizados y llevan a cabo, sobre todo, actividades interpretativo-educativas guiadas. Los visitantes que desarrollan actividades deportivo-aventureras son más especializados y su presencia se ha detectado básicamente en el Parque Natural del Turia. Presentan un perfil y rasgos de personalidad diferentes a los de otros parques. No obstante, muestran también un elevado grado de satisfacción con la visita.

Se puede además concluir que, pese a las valoraciones positivas sobre la experiencia recreativa, el estudio de los cuatro espacios naturales protegidos a partir de la observación participante, nos permite sugerir que se puede mejorar el nivel confort psicológico de los visitantes potenciando el conocimiento emocional.

\section{AGRADECIMIENTOS}

Las autoras de este trabajo quieren agradecer a Amparo Sancho y Diego López Olivares la lectura crítica que han realizado de este trabajo y a todos los gestores y personal de los parques que han facilitado el desarrollo de esta investigación.

\section{BIBLIOGRAFÍA}

AENOR (2008): UNE 187002. Disponible en http://www.aenor.es/DOCUMENTOS/ NORMALIZACION/NORMASNACIONALES/EXTRACTOS/(EX)UNE_187002 $=2008 . p d f$ 
ANDRADE, M.J. (2012): «La interpretación de la realidad del destino por parte de los turistas: evaluaciones cognitivas y afectivas». PASOS. Revista de Turismo y Patrimonio Cultural, no 10 (5), 477-494.

BAKER, D.A. y CROMPTON, J.L. (2000): «Quality, satisfaction and behavioral intentions». Annals of Tourism Research, $\mathrm{n}^{\circ} 27$ (3), 785-804.

BALOGLU, S. y McCLEARY, K.W. (1999): «A model of destination image formation». Annals of Tourism Research, $\mathrm{n}^{\circ} 26$ (4), 868-897.

BERNARDA, L. A. y LEWIS, G. (2003): «Informal work and common mental disorders». Social Psychiatry and Psychiatric Epidemiology, $\mathrm{n}^{\circ} 38$ (9), 485-489.

CONELL, J. (2009): «Birdwatching, Twitching and Tourism: towards an Australian perspective». Australian Geographer, no 40 (15), 203-217.

CORBETT, J. B. (2006): Communicating Nature: How we create and understand environmental messages. Island Press.

DAVIDSON, J. (ed.) (2003): Phobic geographies. The phenomenology and spatiality of identity. Ashgate Publishing Co. (Burlington, USA).

DAY, R.L. (1984) : «Modeling Choices Among Alternative Responses to Dissatisfaction ». Advances in Consumer Research, $\mathrm{n}^{\circ} 11,496-499$.

DEVESA, M.; LAGUNA, M. y PALACIOS, A. (2010): «The role of motivation in visitor satisfaction: Empirical evidence in rural tourism». Tourism Management, $\mathrm{n}^{\circ} 31$ (4), 547-552.

ECHTNER, C.M. y RITCHIE, J.R.B. (1991): «The meaning and measurement of destination image». The journal of Tourism Studies, $\mathrm{n}^{\circ} 2$ (2), 2-12.

ENGEL, J.F.; BLACKWELL, R.D. y MINIARD, P.W. (1993): Consumer Behaviour. Dryden Press (Chicago).

EROSKI-CONSUMER (2012): Guía práctica. Parques Naturales, Parques Nacionales y Reservas de la Biosfera. Disponible en http://parquesnaturales.consumer.es/.

FAULLANT, R.; MATZLER, K. y FULLER, J. (2008): «The impact of satisfaction and image on loyalty: The case of alpine ski resorts». Managing Service Quality, $\mathrm{n}^{\circ} 18$ (2), 163-178.

FELDMAN, R. (1999): Psicología. Ed. Mc Graw Hill (México D.F.).

FORNELL, C. (1992): «A national customer satisfaction barometer. The Swedish experience». Journal of Marketing, $\mathrm{n}^{\circ}$ 56, 6-21.

GARTNER, W.C. (1993): «Image formation process». Journal of Travel and Tourism Marketing, $\mathrm{n}^{\circ} 2$ (2-3), 191-215.

GÓMEZ MARTÍN, M.B. (2005): «Reflexión Geográfica en torno al binomio ClimaTurismo». Boletín de la Asociación de Geógrafos Españoles, no 40, 111-134.

GUYER, G. y POLLAND, J. (1997): «Cruise visitor impression of the environment of the Shannon-Erne Waterways system». Journal of Environmental Management, $\mathrm{n}^{\mathrm{o}} 51$, 199-215.

HALL, E.T. (1963): «A system for the notation of Proxemic behavior». American Anthropologist, $\mathrm{n}^{\mathrm{o}} 65$ (1), 1003-26.

HALSTEAD, D.; HARTMAN, D. y SCHIMIDT, S.L. (1994): «Multisource Effects on the Satisfaction Formation Process». Journal of the Academy of Marketing Science, $\mathrm{n}^{\mathrm{o}} 22$, 114-129. 
HSU, C.H.C.; CAI, L.A. y Li, M. (2010): «Expectation, motivation, and attitude: A tourist behavioral model». Journal of Travel Research, $\mathrm{n}^{\circ} 49$ (3), 282-296.

HUNT, J.D. (1975): «Image as a Factor in Tourism Development». Journal of Travel Research, $\mathrm{n}^{\circ} 13,1-7$.

HVENEGAARD, G.T. (2002): «Using Tourist Typologies for Ecotourism Research». Journal of Ecotourism, $\mathrm{n}^{\mathrm{o}} 1$ (1), 7-18.

KIM, S.S.; SCOTT, D. y CROMPTON, J.L. (1997): «An exploration of the relationships among social psychological involvement, behavioral involvement, comminment, and future intentions in the context of birdwatching». Journal of Leisure Research, $\mathrm{n}^{\circ} 29$ (3), 320-341.

KOTLER, P.; HAIDER, D.H. y REIN, Y. (1993): Marketing places: attracting investment, industry and tourism to cities, states and nations. The free Press (New York).

LEPP, A. y GIBSON, H. (2003): «Tourist roles, perceived risk and international tourism». Annals of Tourism Research, $\mathrm{n}^{\mathrm{o}}$ 30, 606-624.

LINDHAGEN, A. (1996): Forests Recreation in Sweden. Four Case Studies using Quantitative and Qualitative Methods. Dissertation, Swedish University of Agricultural Science.

LINE, N. y COSTEN, W. (2011): Environmental attitudes, motivation, and attachment: Toward a model of nature-based tourism. International CHRIE Conference-Refereed Track. Paper 5.

LUO, Y. y DENG, J. (2008). «The new environmental paradigm and nature-based tourism motivation». Journal of Travel Research, $\mathrm{n}^{\circ}$ 46, 392-402.

MANNING, R.E. (1999): Studies in outdoor recreation: Search and research for satisfaction. Oregon State University Press (Corvallis).

MARION, J.L. y FARRELL, T.A. (1998): «Managing ecotourism visitation in protected areas» en Ecotourism: A Guide for Planners and Managers (Lindberg, Wood y Engeldrum, eds.). The Ecotourism Society (North Bennington, VT), 155-181.

MASLOW, A.H. (1954): Motivation and personality. Harper y Row (New York).

MAYO, E.J. y JARVIS, L.P. (1981). The psychology of the leisure travel: Effective marketing and selling of travel services. CBI Pub. Co. (Boston, Mass.).

McCOOL, S.F. (1983): «The National Parks in Post-Industrial America». Western Wildlands, n 9 (2), 14-19.

MORANT, M. (2007): Desarrollo de un modelo para la determinación de la capacidad de carga recreativa y su aplicación espacios naturales protegidos de la Comunidad Valenciana. Tesis Doctoral. Universidad Politécnica de Valencia.

MORANT, M. y VIÑALS, M.J. (2010). «Modelo para evaluar la capacidad de carga recreativa en áreas de uso intensivo de espacios protegidos. Casos de estudio de la Comunidad Valenciana (España)» en Turismo y gestión de espacios protegidos. Tirant lo Blanc (Valencia), 620-636.

MORGAN, D.J. y LOK, L. (2000): «Assessment of a Comfort Indicator for Natural Tourist Attractions: The Case of Visitors to Hanging Rock, Victoria». Journal of Sustainable Tourism, $\mathrm{n}^{\circ} 8$ (5), 393-408.

MOSCARDÓ, G. (1996): «Mindful Visitors: Heritage and Tourism». Annals of Tourism Research, $\mathrm{n}^{\circ} 23$ (2), 376-397.

OLGYAY, V. (1998): Arquitectura y Clima. Manual de diseño bioclimático para arquitectos y urbanistas. Ed. Gustavo Gili. 
OLIVER, R.L. (1993): «Cognitive, Affective, and Attribute Bases of the Satisfaction Response». Journal of Consumer Research, $\mathrm{n}^{\circ}$ 20, 418-430.

PIKE, S. y RYAN, C. (2005): «Destination positioning analysis through a comparison of cognitive, affective and conative perceptions». Journal of Travel Research, $\mathrm{n}^{\circ} 42$ (4), 333-342.

PIZAM, A. y MANSFELD, Y. (eds.) (1999): Consumer Behavior in Travel and Tourism. The Haworth Hospitality Press (New York).

PLOG, S.C. (1974): Why destinations areas rise and fall in popularity. Cornell Hotel and Restaurant Quarterly, $\mathrm{n}^{\mathrm{o}} 14$ (4), 55-58.

POPE, H.G.; PHILLIPS, K.A. y OLIVARDIA, R. (2001): The Adonis Complex: the secret crisis of male body obsession. Bargain Books (New York).

RODGER, K.; MOORE, S.A. y TAPLIN, R. (2012): Visitor satisfaction, loyalty and protected areas: a review and the future. Informe WA Department of Environment and Conservation, Parks Victoria and the Parks Forum. Technical Report, Murdoch University (Murdoch WA).

ROOVERS, P.; HERMY, M. y GULINK, H. (2002): «Visitor profile, perceptions and expectations in Forests from a gradient of increasing urbanization in central Belgium». Landscape Urban Planning, n 59, 129-145.

SCOTT, D. y THIGPEN, J. (2003): «Understanding the Birder as Tourist: Segmenting Visitors to the Texas Hummer/Bird Celebration». Human Dimensions of Wildlife, $\mathrm{n}^{\circ} 8$, 199-218.

SHIFFMANN, L.G. y LAZAR KANUK, L. (2000): Consumer Behavior. Prentice Hall.

SLATER, K. (1985): Human comfort. Charles C. Thomas Publisher (Springfield, IL).

SÖNMEZ, S. (1998): «Tourism, terrorism and political instability». Annals of Tourism Research, $\mathrm{n}^{\circ} 25,416-456$.

SÖNMEZ, S. y GRAEFE, A. (1998): «Influence of terrorism risk on foreign tourism decisions». Annals of Tourism Research, $\mathrm{n}^{\circ} 25,112-144$.

SPRENG, R.A.; MacKENZIE, S.B. y OLSSHVSKY, R.W. (1996): «A re-examination of the determinants of consumer satisfaction». Journal of Marketing, $\mathrm{n}^{\circ}$ 60, 15-32.

SWAN, J.E. y OLIVER, R.L. (1989): «Postpurchase communications by consumers». Journal of Retailing, $\mathrm{n}^{\circ} 65$ (2), 516-33.

TIAN-COLE, S. y CROMPTON, J.L. (2003): «A conceptualization of the relationships between service quality and visitor satisfaction, and their links to destination selection». Leisure Studies, $\mathrm{n}^{\mathrm{o}} 22$ (1), 65-80.

UNITED STATES DEPARTMENT OF AGRICULTURE FOREST SERVICE (2012): Recreation, heritage and wilderness programs. National visitor use monitoring program. Disponible en http://www.fs.fed.us/recreation/programs/nvum/.

VAN TILBURG, M.L. y VINGERHOETS, J.J.M. (eds.) (2005): Psychological Aspects of Geographical Moves. Homesickness and Acculturation Stress. University Press (Amsterdam).

VASKE, J.J. y DONNELLY, M.P. (2002): «Generalizing the encounter-norm-crowding relationship». Leisure Sciences, $\mathrm{n}^{\circ}$ 24, 255-269.

VIÑALS, M.J.; MORANT, M.; HERNÁNDEZ, C.; FERRER, C.; QUINTANA, R. D.; MARAVALL, N. CABRELLES, G. RAMIS, J. y BACHILLER, C. (2004): «Albufera de 
Valencia (Spain): Measuring carrying capacity in a fragile ecosystem» en Indicators of sustainable development for tourism destinations: A Guidebook. World Tourism Organization (Madrid), 330-337.

VIÑALS, M.J.; Z. HALASA, Z.; ALONSO-MONASTERIO, M. (2011): «Visitors profile of a Mediterranean green corridor. Case study of the Parc Fluvial del Turia (Valencia, Spain)» en Research Studies on Tourism and Environment. Nova Science Publishers (New York), 57-67.

VIVAS, G. F. (2009): «El espacio público como parte del sistema de lugares en tres casos de estudio». Fermentum, no 54, 11-34.

WANG, X.; ZHANGE, J.; GU, C. y ZHEN, F. (2009): «Examining antecedents and consequences of tourist satisfaction: A structural modeling approach». Tsinghua Science and Technology, $\mathrm{n}^{\circ} 14$ (3), 397-406.

WILSON, E.O. (1986): Biophilia. Harvard University Press.

WORLD TOURISM ORGANIZATION (2004). Tourism Congestion Management at Natural and Cultural Sites. UNTWO (Madrid).

WORLD TOURISM ORGANIZATION (2005). Indicators of sustainable development for tourism destinations. Guidebook. UNWTO (Madrid).

XUEQUING QI, CH.; GIBSON, H.J. y ZHANG, J.J. (2009): «Perceptions of Risk and Travel Intentions: The Case of China and the Beijing Olympic Games». Journal of Sport \& Tourism, $\mathrm{n}^{\mathrm{o}} 14$ (1), 43-67.

YUKSEL, A.; YUKSEL, F. y BILIM, Y. (2010): «Destination attachment: Effects on customer satisfaction and cognitive, affective and conative loyalty». Tourism Management, $n^{\circ} 31,274-284$.

ZABKAR, V.; BRENCIC, M. y DMITROVIC, T. (2010): «Modelling perceived quality, visitor satisfaction and behavioural intentions at the destination level». Tourism Management, $\mathrm{n}^{\mathrm{o}} 31$ (4), 537-546.

ZUCKERMAN, M. (2007): Sensation seeking and risky behavior. American Psychological Association (Washington D.C.). 